\title{
Isolated tuberculosis of the wrist: A rare case of extrapulmonary tuberculosis
}

\author{
Mohamed Altayeb Mussa, Edmund Fitzgerald O'Connor, \\ Stuart Waterston, Michael Taylor, Fortune Iwuagwu
}

\begin{abstract}
Introduction: Atypical infections with mycobacteria are unusual in the developed countries and tuberculous involvement of the wrist or carpal bones is a rare presentation. Case Report: This is a new rare case of tuberculosis of the wrist joint in a non-immunocompromised 24-year-old male without concomitant pulmonary tuberculosis. He then underwent two months course of intra-articular steroid injections and methotrexate therapy for a presumed diagnosis of inflammatory oligoarthritis of the wrist as radiographs were reported as normal. The diagnosis was initially obscured by lack of systemic symptoms and was established by direct visualization of acid-fast bacilli on joint fluid and biopsy of the abscess. Musculoskeletal involvement in tuberculosis may be easily missed because of its non-specific clinical signs. The disease may mimic inflammatory arthritis and high index of suspicion is required when dealing with long standing inflammatory swellings.
\end{abstract}

Mohamed Altayeb Mussa1, Edmund Fitzgerald O'Connor', Stuart Waterston ${ }^{2}$, Michael Taylor ${ }^{3}$, Fortune Iwuagwu ${ }^{4}$ Affiliations: ${ }^{1}$ Orthopaedics Specialist Registrar, Hull Royal Infirmary, 4 School Lane Mews, Beverley, East Riding of Yorkshire, HU17 9LS, United Kingdom; 'Plastic Surgery Specialist Registrar, Mid Essex Hospital, Court Road, Broomfield, Chelmsford, Essex, CM1 7ET, United Kingdom; ${ }^{3}$ Consultant Orthopaedics and Traumatology, Mid Essex Hospital, Court Road, Broomfield, Chelmsford, Essex, CM1 7ET, United Kingdom; ${ }^{4}$ Consultant Hand and Plastic surgery, Mid Essex Hospital, Court Road, Broomfield, Chelmsford, Essex, CM1 7ET, United Kingdom.

Corresponding Author: Mohamed Altayeb Mussa, Orthopaedics Specialist Registrar, Hull Royal Infirmary, 4 School Lane Mews, Beverley, East Riding of Yorkshire, HU17 9LS, United Kingdom; Tel: 00447590334551; Email: mohamedaltayeb@ gmail.com

Received: 11 February 2013

Accepted: 10 May 2013

Published: 01 October 2013
Conclusion: Persistent swelling of bones or joints can be a presenting sign of tuberculosis. A normal chest radiograph or the absence of systemic symptoms does not exclude the possibility of bone tuberculosis. When confronted with unusual inflammatory findings, always send tissue for histology and alcohol and acid-fast bacilli (AAFB) culture.

Keywords: Tuberculosis, Wrist, Oligoarthritis, Carpal bones

$$
* * * * * * * * *
$$

Mussa MA, O'Connor EF, Waterston S, Taylor M, Iwuagwu F. Isolated tuberculosis of the wrist: A rare case of extrapulmonary tuberculosis. International Journal of Case Reports and Images 2013;4(10):541-545.

$* * * * * * * * *$

doi:10.5348/ijcri-2013-10-375-CR-4

\section{INTRODUCTION}

There has been a significant worldwide increase in the prevalence of tuberculosis (TB) and of its extrapulmonary manifestations $[1,2]$. Osteoarticular TB accounts for $1-2 \%$ of all the TB cases in the western world [3]. The spine being the most common site of osseous involvement, accounting for about 50\% of cases [4]. Tuberculous involvement of the wrist joint is a rare presentation. The diagnosis may be initially difficult owing to the rarity of the disease, its non-specific clinical signs and lack of awareness resulting in delay in starting treatment, progression of disease and more advanced disability [5].

\section{CASE REPORT}

A previously healthy 24-year-old British male was admitted to the accident and emergency department 
with a facial laceration after being involved in a fight. An incidental finding of a swollen right wrist was made and was subsequently referred to our hand trauma and plastic surgery unit with a diagnosis of an abscess. The patient gave a one-year history of a spontaneously appearing painful lump on the dorsum of the right dominant hand, which had gradually increased in size with no history of trauma. There was no associated history of fever, loss of weight, night sweats or fatigue.

Prior to presentation, he had been initially treated with non-steroidal anti-inflammatories for a painful wrist joint. He then underwent two months course of intraarticular steroid injections and methotrexate therapy for a presumed diagnosis of inflammatory oligoarthritis of the wrist as radiographs were reported as normal. The condition did not improve and wrist movements became increasingly painful and restricted.

Clinical examination revealed an average built man with no peripheral lymphadenopathy. There was a painless swelling located on the dorso-radial aspect of the right wrist, measuring approximately $4 \times 4 \mathrm{~cm}$ with cellulitic inflamed overlying skin (Figure 1). The patient was very reluctant to actively move his affected wrist and passive range of movement was limited by pain. The rest of the clinical examination including respiratory system examination was unremarkable. Blood tests showed hemoglobin $15.3 \mathrm{~g} / \mathrm{dL}$, total leukocyte count $8,900 / \mathrm{mm}^{3}$, neutrophils $5.2 \times 10^{3} / \mathrm{L}$, lymphocytes $1.0 \times 10^{9} /$ $\mu \mathrm{L}$, monocytes $0.9 \times 10^{3} / \mathrm{L}$, eosinophil $\mathrm{o} / \mu \mathrm{L}$, erythrocyte sedimentation rate $32 \mathrm{~mm} / \mathrm{h}$ and C-reactive protein $5.0 \mathrm{mg} / \mathrm{L}$. He was HIV seronegative and chest radiograph was normal with no signs of pulmonary tuberculosis (Figure 2). Computed tomography (CT) scan (Figure 3) and radiographs of the right hand and wrist AP and lateral views (Figures 4 and 5) revealed multiple carpal and distal radius erosions. Magnetic resonance imaging (MRI) scan with contrast confirmed the presence of multiple osteolytic lesions and showed extensive soft tissue thickening, edema of the extensor tendons and fluid noted affecting mainly the dorsum of the wrist (Figure 6).

Drainage of the wrist yielded pus and inflammatory tissue. Histological assessment showed caseating granuloma, whilst staining confirmed the presence of acid-fast bacilli. The diagnosis of extrapulmonary tuberculosis affecting the distal radius and carpal bones with tuberculous tenosynovitis was made. Antituberculous treatment was commenced with four drugs (rifampicin, ethambutol, isoniazid and pyrazinamide) for two months, followed by two drugs (rifampicin, isoniazid) for 12 months. The infection settled with antitubercular drugs and no further intervention was needed.

\section{DISCUSSION}

Tuberculous infection of the musculoskeletal system is rare even in areas of high TB prevalence [6]. Hand

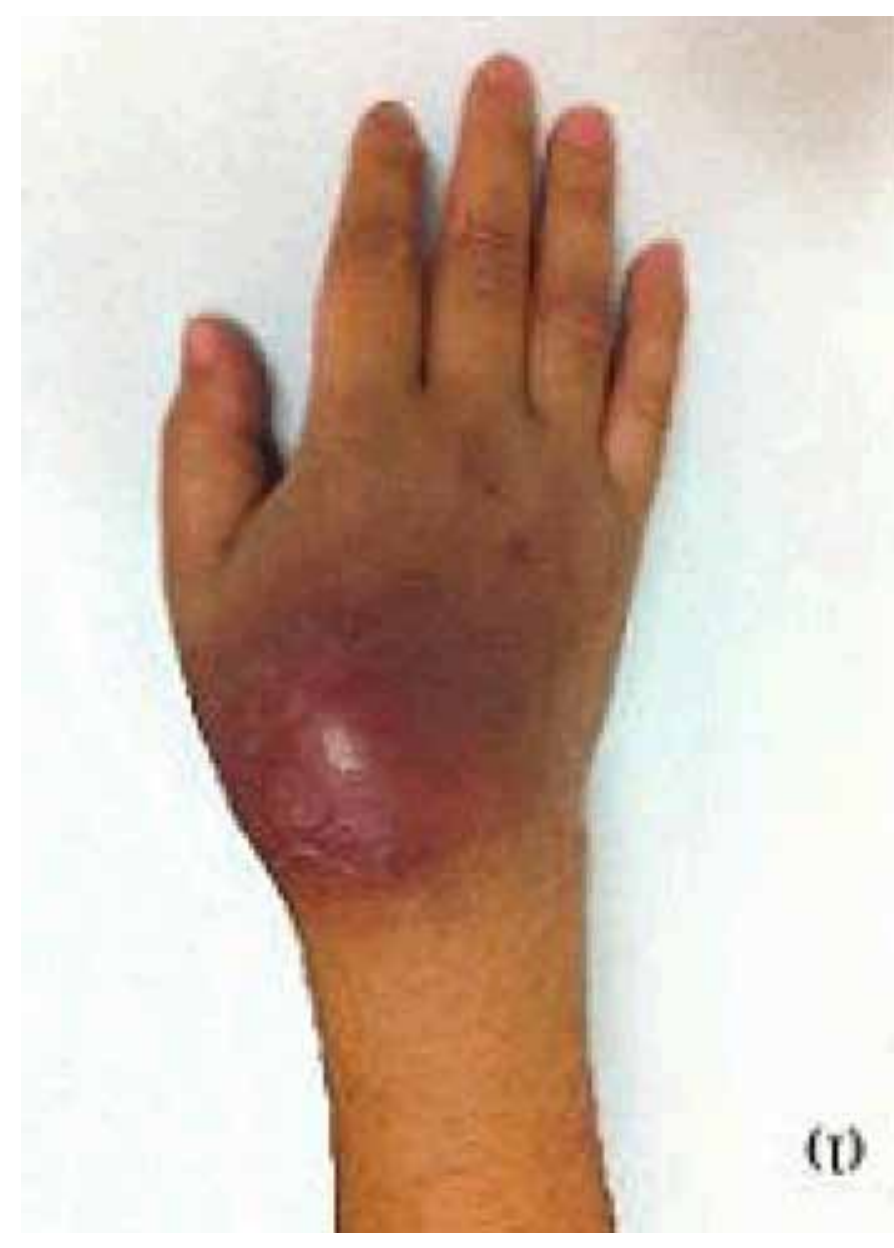

Figure 1: Swelling of dorsum of right hand and wrist joint with cellulitic overlying skin.

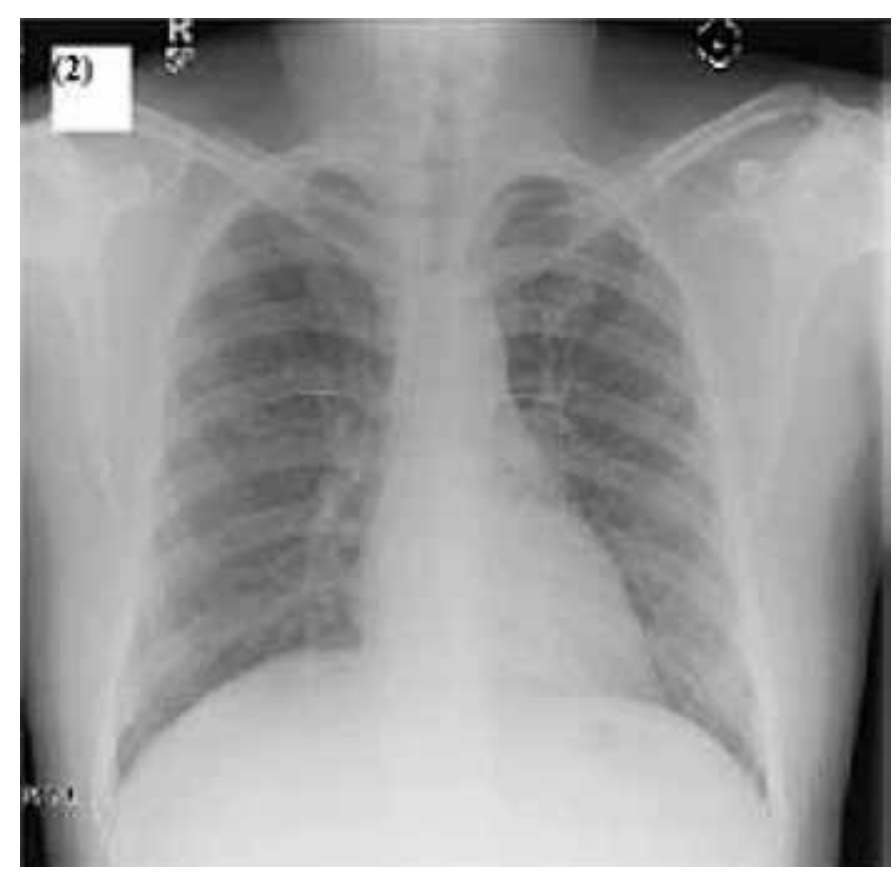

Figure 2: Chest X-ray showing no lesions. 


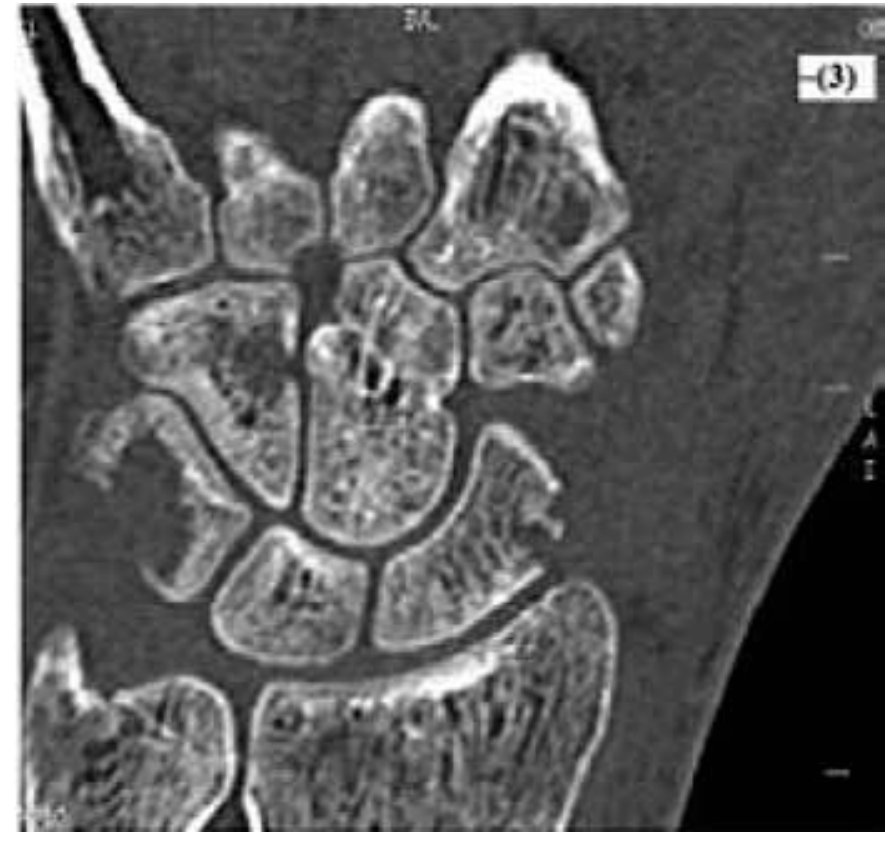

Figure 3: Computed tomography scan of wrist and carpal bones showing extensive multiple erosions of carpal bones and adjacent radial styloid.

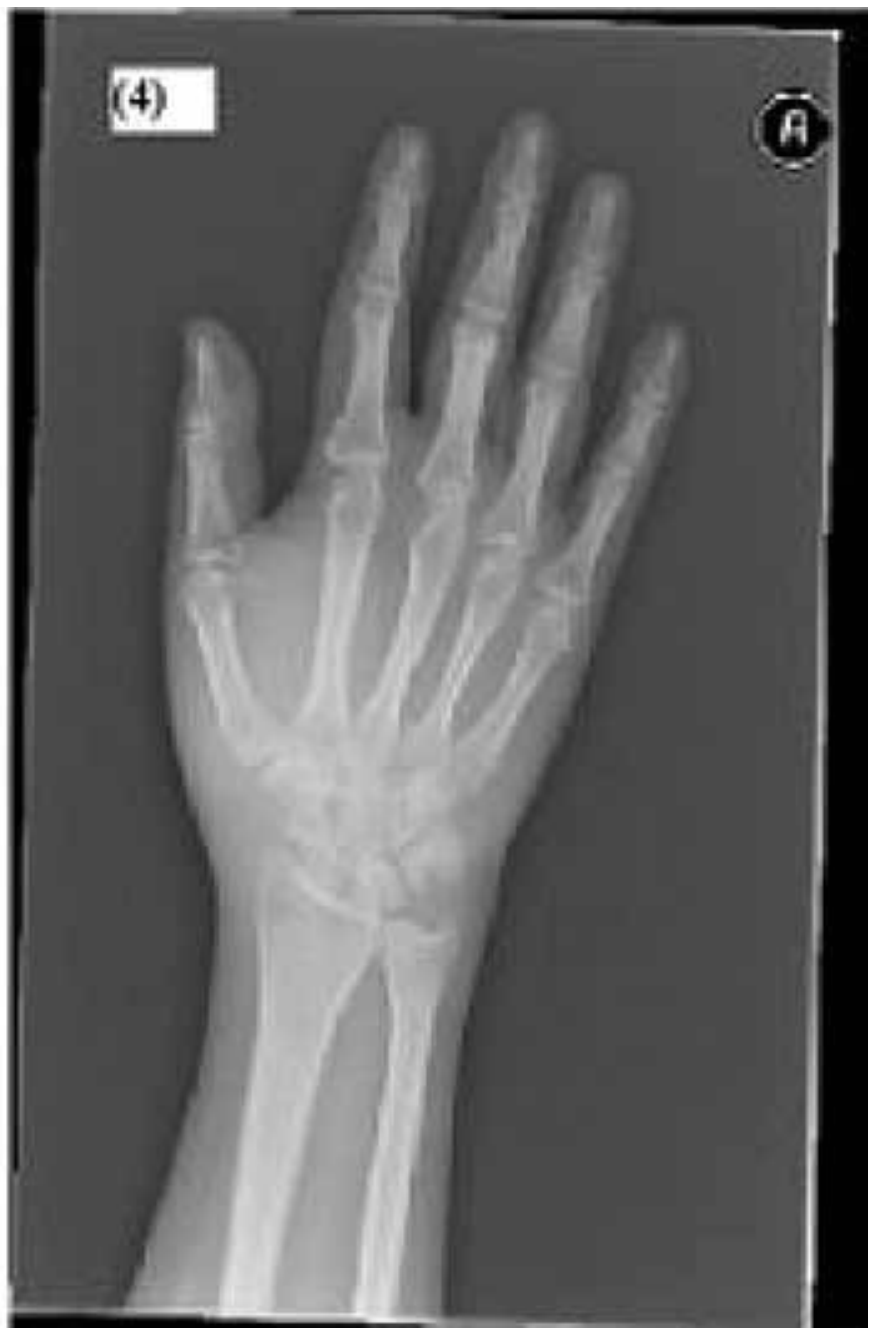

Figure 4: X-ray of right hand and wrist joint (AP) projection showing erosions of carpal bones.

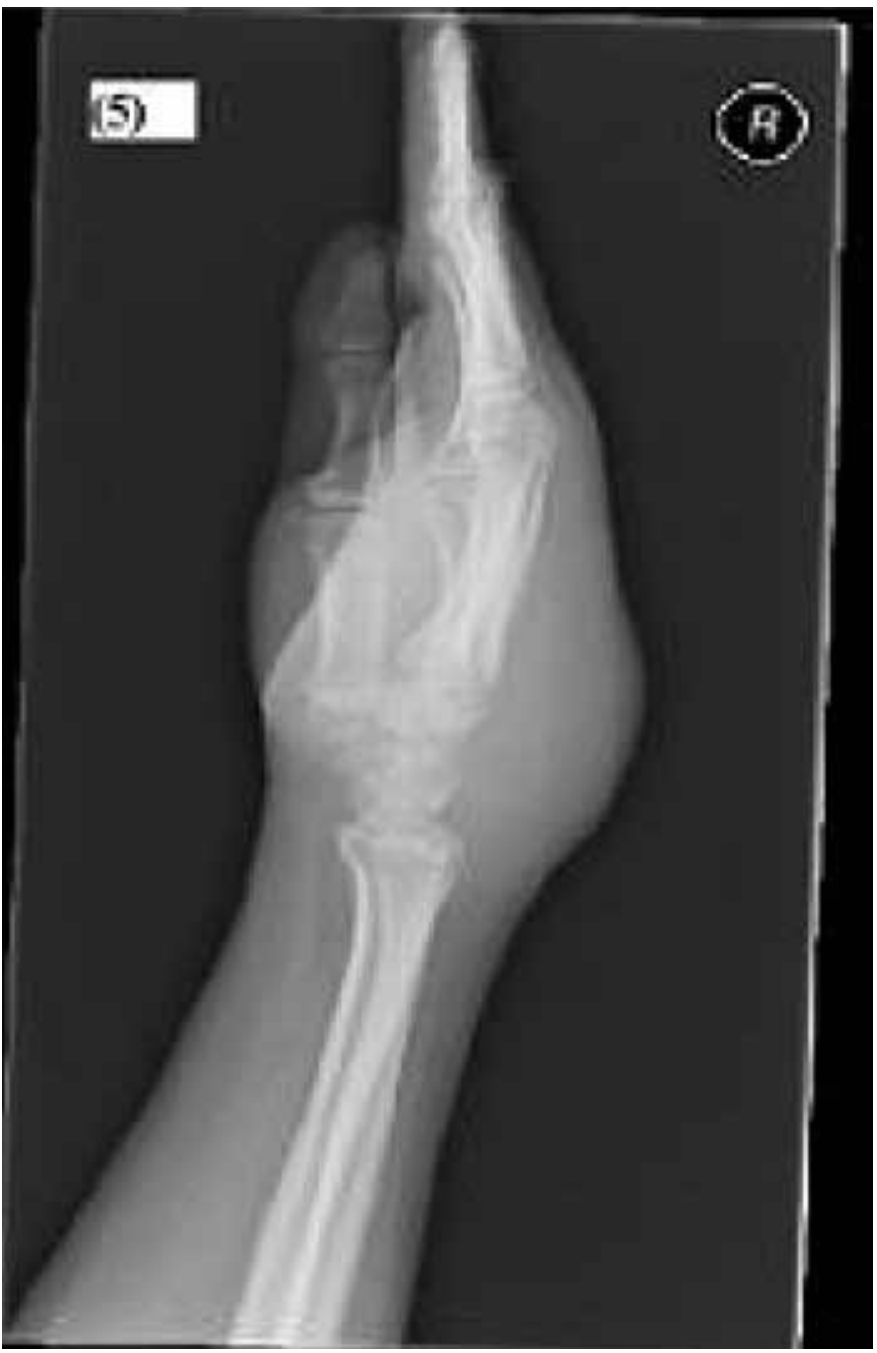

Figure 5: X-ray of right hand and wrist lateral projection.

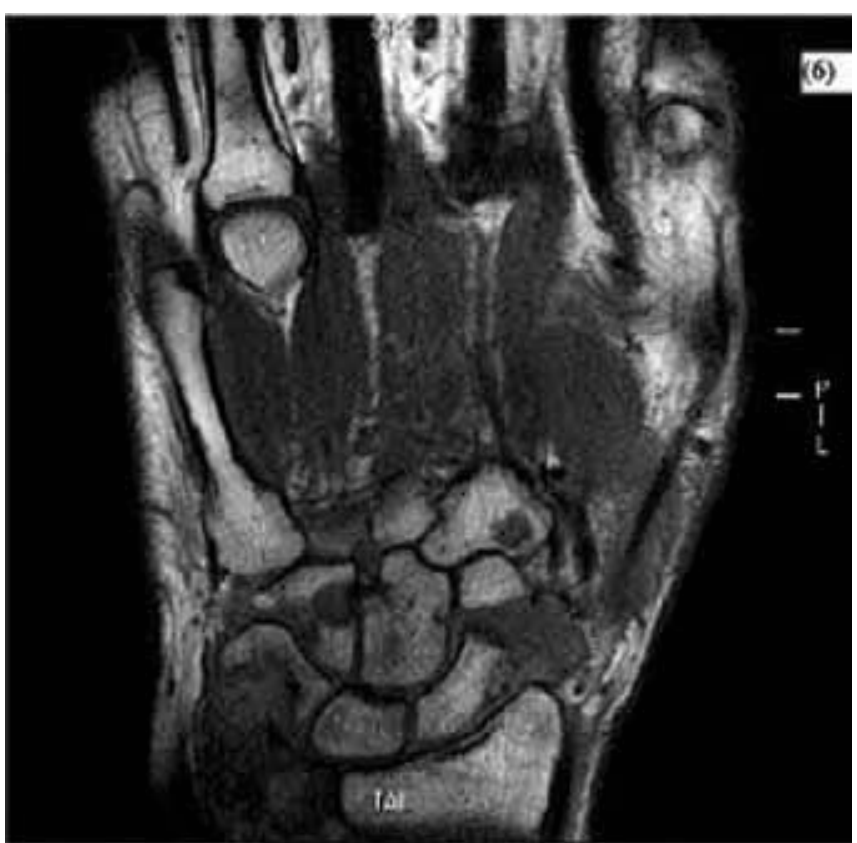

Figure 6: Magnetic resonance imaging scan of right hand and wrist with contrast showing extensive soft tissue thickening, edema and fluid around the extensor tendons and also to the common flexor sheath in the wrist. Multiple erosions are seen in the carpal bones and the MCP bases. 
and wrist involvement are seen in $10 \%$ of patients with musculoskeletal disease [7]. The symptoms initially are non-specific in the form of joint pain, swelling, effusion, stiffness, carpal tunnel syndrome, limitation of movement, and discharging sinuses. Constitutional symptoms include low-grade fever, night sweats, weight loss, and anorexia. Blood tests may show mildly raised or even normal inflammatory markers.

Sclerosis and osteolytic lesions, the main radiographic features of bony tuberculosis, are non-specific and are present in other conditions such as inflammatory arthritis, pyogenic osteomyelitis, and some malignancies [8]. Other radiographic features include osteopenia, soft-tissue swellings, periosteal reaction, narrowing of the joint space and bone cysts [8-10]. The CT and MRI scans, although non-specific, may help in the differential diagnosis and in evaluating the extent of the lesion. The diagnosis of TB may be confirmed on the recognition of Mycobacterium tuberculosis on either histology study or culture, or, ideally, both [11-13]. But the gold standard for the diagnosis of osseous tuberculosis would be culture of Mycobacterium tuberculosis from bone tissue.

There is no consensus on the accurate duration of treatment and this remains a matter of great debate. However, a prolonged course of antituberculous drugs is the basis of treatment $[5,7,14]$. Watts and Lifeso recommended that treatment should be continued for a minimum of 12 months for osteoarticular involvement [15]. Kotwal and Khan [16] treated cases of hand tuberculosis with antitubercular chemotherapy using four drugs (INH, rifampin, pyrazinamide, and ethambutol) for four months; followed by three drugs (INH, rifampin, and pyrazinamide) for three months; and finally two drugs (INH and rifampin) for 11 months [17]. However, the optimal duration of treatment has been an issue of considerable debate.

The absence of active pulmonary tuberculosis does not eliminate the possibility of an osteoarticular tuberculosis [18]. Systemic disease, diabetes mellitus, and local factors such as trauma or intra-articular steroids may predispose to activation of a distant focus [9]. The clinical picture and radiological features in the early stages of the disease may simulate arthritis and a definitive diagnosis is established by open biopsy and tissue culture [10]. Surgery is an adjunct to the antituberculosis medications as surgical procedures may be necessary to control the disease or to improve the function.

\section{CONCLUSION}

In summary, persistent swelling of bones or joints can be a presenting sign of tuberculosis. The diagnosis can be easily missed because of the non-specific clinical signs resulting in progression of disease and more advanced disability. The disease may mimic inflammatory arthritis and therefore, high index of suspicion is required when dealing with long standing inflammatory swellings not responding to conservative measures. A normal chest radiograph or the absence of systemic symptoms does not exclude the possibility of bone tuberculosis. When confronted with unusual inflammatory findings, always send tissue for histology and alcohol and acid-fast bacilli culture. Surgery is an adjunct to drugs to control the disease or to improve the function. Because results are much better during earlier stages of the disease, prompt diagnosis and adequate treatment are essential.

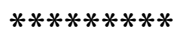

\section{Author Contributions}

Mohamed Altayeb Mussa - Substantial contributions to conception and design, Acquisition of data, Analysis and interpretation of data, Drafting the article, Revising it critically for important intellectual content, Final approval of the version to be published

Edmund Fitzgerald O'Connor - Acquisition of data, Analysis and interpretation of data, Drafting the article, Revising it critically for important intellectual content, Final approval of the version to be published

Stuart Waterston - Acquisition of data, Revising it critically for important intellectual content, Final approval of the version to be published

Michael Taylor - Acquisition of data, Revising it critically for important intellectual content, Final approval of the version to be published

Fortune Iwuagwu - Acquisition of data, Revising it critically for important intellectual content, Final approval of the version to be published

\section{Guarantor}

The corresponding author is the guarantor of submission.

\section{Conflict of Interest}

Authors declare no conflict of interest.

\section{Copyright}

(C) Mohamed Altayeb Mussa et al. 2013; This article is distributed under the terms of Creative Commons attribution 3.0 License which permits unrestricted use, distribution and reproduction in any means provided the original authors and original publisher are properly credited. (Please see www.ijcasereportsandimages.com/ copyright-policy.php for more information.)

\section{REFERENCES}

1. Barritt AW, Clark L, Teoh V, Cohen AM, Gibb PA. Assessing the adequacy of procedure-specific consent forms in orthopaedic surgery against current methods of operative consent. Annals of the Royal College of Surgeons of England 2010 Apr;92(3):246-9.

2. Evanchick CC, Davis DE, Harrington TM. Tuberculosis of peripheral joints: An often missed diagnosis. The Journal of Rheumatology 1986 Feb;13(1):187-9. 
3. Shah BA, Splain S. Multifocal osteoarticular tuberculosis. Orthopedics 2005 Mar;28(3):329-32.

4. Moon MS. Tuberculosis of the spine. Controversies and a new challenge. Spine 1997 Aug 1;22(15):1791-7.

5. Subasi M, Bukte Y, Kapukaya A, Gurkan F. Tuberculosis of the metacarpals and phalanges of the hand. Annals of plastic surgery 2004 Nov;53(5):46972 .

6. Leung PC. Tuberculosis of the hand. The Hand 1978 Oct;10(3):285-91.

7. Martini M, Benkeddache Y, Medjani Y, Gottesman H. Tuberculosis of the upper limb joints. International orthopaedics 1986;10(1):17-23.

8. Agarwal S, Caplivski D, Bottone EJ. Disseminated tuberculosis presenting with finger swelling in a patient with tuberculous osteomyelitis: A case report. Annals of clinical microbiology and antimicrobials 2005;4:18.

9. Berney S, Goldstein M, Bishko F. Clinical and diagnostic features of tuberculous arthritis. The American journal of medicine $1972 \mathrm{Jul} ; 53(1): 36-42$.

10. Wallace R, Cohen AS. Tuberculous arthritis: A report of two cases with review of biopsy and synovial fluid findings. The American journal of medicine 1976 Aug;61(2):277-82.
11. Karanas YL, Yim KK. Mycobacterium tuberculosis infection of the hand: A case report and review of the literature. Annals of plastic surgery 1998 Jan;40(1):65-7.

12. Watts HG, Lifeso RM. Tuberculosis of bones and joints. J Bone Joint Surg Am 1996 Feb;78(2):288-98.

13. Dhillon MS, Gupta RK, Bahadur R, Nagi ON. Tuberculosis of the sternoclavicular joints. Acta orthopaedica Scandinavica 2001 Oct;72(5):514-7.

14. Skoll PJ, Hudson DA. Tuberculosis of the upper extremity. Annals of plastic surgery 1999 Oct;43(4):374-8.

15. St Clair Strange FG. Current concepts review. Tuberculosis of bones and joints (78-A:288-298, Feb. 1996) by Watts and Lifeso. J Bone Joint Surg Am 1998 Apr;80(4):604.

16. Kotwal PP, Khan SA. Tuberculosis of the hand: Clinical presentation and functional outcome in 32 patients. J Bone Joint Surg Br 2009 Aug;91(8):10547.

17. Al-Qattan MM, Al-Namla A, Al-Thunayan A, AlOmawi M. Tuberculosis of the hand. J Hand Surg Am 2011 Aug;36(8):1413-21.

18. Mann KJ. Lung lesions in skeletal tuberculosis; review of 500 cases. Lancet 1946 Nov 23;2(6430):744-9.
Access full text article on other devices

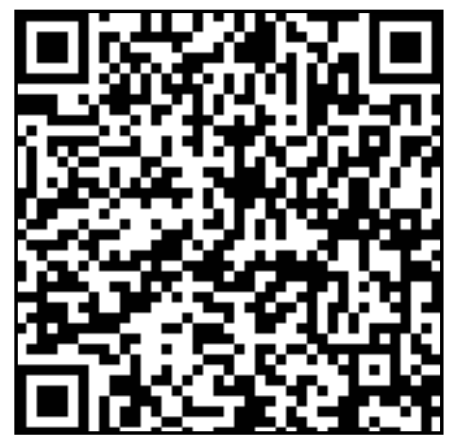

Access PDF of article on other devices

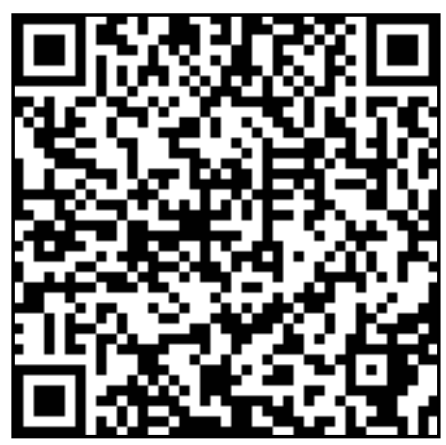

